\title{
Prática de Avaliação: a construção de pareceres avaliativos em uma disciplina oferecida em um Mestrado Profissional
}

\author{
Lúcia Regina Goulart Vilarinho \\ Kátia Taucei Perez
}

Sandra Maria Martins Redoválio Ferreira

\section{Resumo}

Este artigo relata uma experiência conduzida na disciplina Prática de Avaliação de um Mestrado Profissional em Avaliação, quando foi solicitado aos alunos que elaborassem um Parecer Avaliativo sobre artigos que tratassem do mesmo tema. Os alunos encontraram 26 artigos que abordavam o tema Material Didático, depositados no período 2001-2016, na base de dados deste mestrado (e-Aval). Os artigos foram, então, analisados quantitativamente em relação aos aspectos: tipo de artigo; classificação por nível educacional; classificação da publicação segundo a região geográfica; e distribuição segundo o tema abordado. Em seguida, passaram por uma análise qualitativa que buscou aprofundar como esses artigos discutiam os principais itens que devem estar presentes em uma publicação dessa natureza, a saber: o problema, o objetivo de estudo, o referencial teórico, a metodologia, os resultados e as recomendações. Nesta análise foram tecidas comentários sobre tais partes, considerando o conjunto dos artigos. As autoras concluíram que ao analisar os 26 artigos da base de dados e-Aval, vinculados à categoria Avaliação de Material Didático, desenvolveram um recorte do Estado da Arte da Avaliação. Esta experiência ilustra o dinamismo da referida disciplina, com a produção de um trabalho cooperativo que contribui para o desenvolvimento crítico e a produção escrita dos alunos.

Palavras-chave. Avaliação. Material Didático. Estado da Arte em Avaliação. Parecer Avaliativo. 


\title{
Evaluation Research Practice: an opportunity for the construction of evaluation opinions
}

\author{
Lúcia Regina Goulart Vilarinho \\ Kátia Taucei Perez \\ Sandra Maria Martins Redoválio Ferreira
}

\section{Abstract}

This article addresses an experience conducted in the research practice course of a professional master's program in evaluation, in which it was asked for the students to elaborate an evaluative report on articles with the same theme as this study. Twenty-six articles that discuss the didactic material theme were found deposited from 2001-2016 in the Master's program database (e-Aval). The articles were analyzed quantitatively according to the following aspects: type of article; classification by educational level; publication classification according to geographic region; and distribution according to the theme discussed. Subsequently, the articles underwent a qualitative analysis in which considerations were made regarding their main parts: problem, study's objective, theoretical framework, methodology, results and recommendations. In this analysis, comments were made on these parts, considering all articles. The authors concluded that by analyzing the 26 articles from the e-Aval database, based on the Evaluating Didactic Material category, a 'cut-off' of the State of the Art of Evaluation was developed. This experience illustrates the dynamism of this discipline, with the production of a cooperative work that contributes to the critical development and written production of students.

Key words: Evaluation. Didactic material. State of the Art of Evaluation. Evaluative report. 


\section{Introdução}

O Programa de Mestrado Profissional em Avaliação da Faculdade Cesgranrio, de cunho multidisciplinar, tem como orientação básica fomentar nos alunos o pensamento analítico e crítico, além de desenvolver competências técnicas relacionadas a esta área. Um dos instrumentos para concretizar esta perspectiva é a oferta, em seu currículo, da disciplina Prática de Avaliação, a qual, entre outros objetivos, solicita aos alunos a construção/atualização de um estado da arte online na referida área.

Batizado como e-Aval, este banco de dados nasceu de um projeto de pesquisa do referido Mestrado, tendo por objetivo investigar, por meio de um processo estruturado de busca em uma base eletrônica de dados, a SciELO, o estado da arte da área da Avaliação (FACULDADE CESGRANRIO, E-AVAL, 2018). A seleção dos artigos para inclusão no e-Aval tem como critérios: a delimitação do campo de pesquisa à área da Educação e a existência dos vocábulos Educação e Avaliação, dentre as palavras-chave e títulos dos artigos.

A organização do e- Aval tem se dado considerando os artigos inseridos na referida base a cada ano. Assim, para o período 2001-2018, esta base já tinha consolidado um total de 839 artigos vinculados à área da avaliação, ficando cada nova turma que participa da disciplina encarregada, sob a orientação de suas docentes, de ampliá-la com os trabalhos mais recentes. Em 2018, além dessa consolidação, foi pedido aos alunos que fizessem um 'Parecer Avaliativo' sobre um conjunto de artigos que tratassem de um mesmo tema.

Entre os 839 artigos catalogados, encontrou-se 26 abordando a categoria 'material didático' e 47 tratando de 'instrumento de avaliação', os quais foram selecionados pelos alunos para serem avaliados com vistas à elaboração do Parecer Avaliativo. Buscando subsidiar os alunos, as docentes da Prática de Avaliação ofereceram um roteiro para a composição do

Parecer, integrado pelas seguintes partes: (a) introdução, na qual seriam apresentados os problemas, os objetivos, as questões dos estudos e a importância dos trabalhos; (b) procedimentos metodológicos, que descreveu como cada artigo abordou a metodologia de seu estudo; (c) resultados ou respostas às questões de estudo; e (d) conclusões, que apresentou as considerações e recomendações encontradas nos artigos. Este estudo relata, pois, a experiência realizada pelo aluno que assumiu realizar o Parecer Avaliativo referente ao conjunto de artigos que integrou a categoria "material didático".

\section{Procedimentos metodológicos}

O critério de escolha dos artigos foi terem nos títulos e nas palavras-chave o termo avalição e educação. Foram encontrados 26 artigos para serem analisados, de acordo com os seguintes aspectos: (a) tipo de artigo; (b) classificação por nível educacional; (c) classificação da publicação segundo a região geográfica; (d) distribuição dos artigos segundo o tema 
abordado. Tratou-se, portanto, de uma análise baseada em questões quantitativas. Em seguida, os artigos passaram por uma análise mais profunda na qual foi verificado se continham determinados aspectos, a saber: objetivo, problema, metodologia, referencial teórico, resultado e recomendação e como tinham sido abordados. A análise desses itens permitiu que se estabelecesse semelhanças e diferenças entre os artigos, em uma dimensão qualitativa.

\section{Análise Inicial}

As tabelas que se seguem oferecem respostas quantitativas às análises iniciais. Assim, no que tange ao tipo de artigo, encontrou-se os dados que estão na tabela 1.

Tabela 1 - Os artigos segundo seu tipo

\begin{tabular}{|l|c|}
\hline \multicolumn{1}{|c|}{ Tipo } & Quantidade de artigos \\
\hline Teórico & 1 \\
\hline Resultado de pesquisa & 18 \\
\hline Relado de experiência & 7 \\
\hline & Total \\
\hline
\end{tabular}

Fonte: as autoras (2018)

A maioria dos artigos (18) se enquadrou no tipo Resultado de Pesquisa, seguida de Relado de Experiência (7) e de apenas um artigo do tipo Teórico. Não é de estranhar que o Resultado de Pesquisa lidere os artigos, pois é o tipo de trabalho que apresenta maior valor para as editorias das revistas acadêmicas. Já o Relato de Experiência, que aparece em segundo lugar, geralmente se refere a um projeto no qual o autor participou e tem como objetivo prático buscar solução para determinado problema ou analisar os resultados da implementação de uma solução.

Em relação à classificação dos artigos segundo o nível educacional, verificou-se que uma parte significativa abordou temas vinculados ao Ensino Superior o que também não representou uma surpresa, pois geralmente são os profissionais dessa área que mais contribuem com artigos em revistas. O Ensino Médio é o segundo nível mais pesquisado. A tabela que se segue oferece uma visão da distribuição dos artigos.

Tabela 2 -Os artigos por níveis educacionais

\begin{tabular}{|l|c|}
\hline \multicolumn{1}{|c|}{ Por nível educacional: } & Quantidade de artigos \\
\hline Educação Básica - Ensino Fundamental & 2 \\
\hline Educação Básica - Fundamental e Ensino Médio & 1 \\
\hline Educação Básica - Ensino Médio & 6 \\
\hline Ensino Superior - Graduação & 15 \\
\hline Educação em geral & 2 \\
\hline Total & 26 \\
\hline
\end{tabular}

Fonte: as autoras (2018) 
Encontrou-se, ainda, que a maioria dos artigos foi produzida por doutores e mestres que têm como objetivo publicar sua produção científica em revistas acadêmicas nacionais. Isto vem ao encontro do que Mancebo (2013, p. 520) esclarece:

[...] os professores de educação superior preparam e ministram cursos na graduação e pós-graduação, orientam estudantes na graduação e na pósgraduação [...], "escolhem" formas de produzir e ter sucesso na publicação de um número importante de artigos em revistas conceituadas no seu respectivo campo de atuação.

Quanto à relação dos artigos com as editorias das revistas, observou-se uma grande concentração de artigos publicados em revistas cujas sedes se localizam na região sudeste. Não se buscou saber de qual região veio o artigo, mas sim em que regiões haviam sido publicados. Este dado também não representa novidade no campo das publicações e se expressa na tabela 3 .

Tabela 3 -Publicações dos artigos por regiões geográficas

\begin{tabular}{|l|c|}
\hline \multicolumn{1}{|c|}{ Por região geográfica: } & Quantidade de artigos \\
\hline Centro-oeste & 1 \\
\hline Nordeste & 2 \\
\hline Sudeste & 19 \\
\hline Sul & 26 \\
\hline Total & 26 \\
\hline
\end{tabular}

Fonte: as Autora (2018)

Foram 19 publicações vinculadas à região sudeste, seguidas de quatro na região sul. Esta informação também não causa qualquer estranheza, pois a maior parte das editoras das revistas acadêmicas situa-se nesta região.

Por fim, a análise voltou-se para determinar as áreas nas quais os artigos foram escritos, tendo se verificado uma predominância da área educacional da saúde, com 21 artigos, seguida de quatro na área da educação. Um artigo foi em educação agrícola. Os temas abordados se encontram na tabela quatro.

Tabela4 - Tipos de material didático

\begin{tabular}{|c|c|}
\hline Temas & Quantidade de artigos \\
\hline Ambiente virtual de aprendizagem & 1 \\
\hline Jogo educacional (tabuleiro) & 1 \\
\hline Livro didático & 3 \\
\hline Mapa conceitual & 3 \\
\hline Matérias televisivas & 1 \\
\hline Objeto virtual & 3 \\
\hline Simulador & 1 \\
\hline Site & 2 \\
\hline Software & 10 \\
\hline WebQuest & 1 \\
\hline Total & 26 \\
\hline
\end{tabular}

Fonte: as Autoras (2018)

ISSN 2526-2882 
Pela Tabela 4 verifica-se que a maior parte dos artigos aborda algum tipo de tecnologia da informação usada como material didático para ajudar na aprendizagem dos alunos, sendo que as mais discutidas foram: uso de software no aprendizado - 10 artigos; e objetos virtuais de aprendizagem, mapas conceituais e livros didáticos - com três artigos em cada categoria.

Esta breve análise quantitativa permitiu que se percebesse a crescente necessidade de se incorporar as tecnologias da informação na educação com vistas a melhorar a formação / formação continuada dos estudantes, uma vez que já fazem parte do dia a dia desses sujeitos. Nesta direção, há que se concordar com Juliani (2009, p. 514) “[...] consideramos que a tecnologia possa estar a favor do professor e do aluno no processo educativo, por combinar estímulos variados, podendo ser menos monótono e ampliar o interesse do aluno". Os softwares foram os mais discutidos, pois representam uma tendência mais antiga na área da avaliação.

\section{Análise Mais Profunda}

Na segunda parte da metodologia, procedeu-se a uma análise mais profunda, a qual incidiu sobre o texto de cada artigo, com a intenção de verificar se apresentavam e como abordavam: o problema, o objetivo de estudo, o referencial teórico, a metodologia, os resultados e recomendações. A seguir são expressos os resultados desta análise.

Existência de Problema - a análise dos artigos levou-nos a inferir que todos traziam um problema ou uma necessidade, às vezes explicitado de maneira mais clara, outras de modo mais difuso. A existência de um problema representa a motivação para a elaboração do estudo. A seguir são transcritos dois exemplos que ilustram a presença de um problema.

$\mathrm{Na}$ última década os computadores de mão se popularizaram entre profissionais da saúde. Nos EUA, por exemplo, cerca de 60-70\% dos estudantes de medicina já os utilizam. A disponibilização de informação clínica nestes dispositivos pode auxiliar no ensino de práticas médicas, no entanto, a maneira como são estruturadas pode influenciar na satisfação e no aprendizado dos alunos. (PINTO, 2010, p. 1)

A informática vem sendo introduzida na educação em ritmo acelerado, obrigando educadores e educandos a familiarizarem-se com essa tecnologia. Este estudo teve como objetivo o desenvolvimento e avaliação do software educacional "A criança e o medicamento". (ZEM-MASCARENHAS; CASSIANI, 2001, p. 13)

Objetivo do Estudo- observou-se que os objetivos dos artigos estavam relacionados ao contexto do estudo. Dos 26 artigos, 22 apresentaram de maneira clara os objetivos, nos demais (quatro) tiveram que ser inferidos, pois só depois de se reler o artigo é que se conseguiu captar o objetivo de estudo. Dois exemplos são aqui registrados, para evidenciar a dificuldade de 
apresentar um objetivo claro.

No estudo que ora apresentamos, dispusemo-nos, pois, a considerar as práticas de numeramento inseridas no âmbito das práticas de letramento, uma vez que nossa análise das atividades de um livro didático voltado para a EJA busca compreender relações que tais atividades supõem, permitem, reforçam ou questionam entre práticas matemáticas escolares e práticas de leitura e escrita de diversos tipos de textos. (ADELINO; FONSECA, 2014, p.185).

O projeto de doutoramento tem como objetivo geral colaborar para o aumento de acesso ao conhecimento sobre a dengue e a doença de Chagas através de tecnologias de informação e comunicação (TIC) por meio do desenvolvimento de CD-ROM.” (PIMENTA et. al, 2008, p.148).

Como se pode verificar, quando não há clareza no objetivo é provável que o autor tenha dificuldade de desenvolver sua metodologia e de obter os resultados do estudo. Em suma, a clareza dos objetivos é fundamental para o desenvolvimento das demais partes do estudo.

Referencial Teórico - verificou-se que os referenciais teóricos dos artigos servem de embasamento para o desenvolvimento do objetivo de estudo e estão baseados em livros, periódicos (físicos ou online) e sites. Foi possível separar os artigos por tipo de material didático para melhor analisar os referenciais teóricos neles contidos, o que ofereceu o seguinte panorama:

(a) cinco artigos falam sobre materiais didáticos: jogo educativo (tabuleiro), matérias televisivas, ambiente virtual, webquest e simulador - apresentam referenciais teóricos únicos, que não aparecem em nenhum outro artigo;

(b) dois artigos abordam o material didático site e têm em comum três referenciais teóricos (um livro e dois artigos);

(c) três artigos falam sobre o material didático mapa conceitual, tendo em comum dois livros;

(d) três artigos falam sobre o material didático livro didático, mas não apresentam autor em comum, embora dois deles citem o Programa Nacional do Livro Didático;

(e) três artigos falam sobre o material didático objeto virtual e usam dois livros em comum;

(f) 10 artigos falam sobre o material didático software e apresentam em comum sete referenciais teóricos (três livros; dois artigos, uma tese de doutorado e uma dissertação);

Ficou, portanto, evidente que em 10 artigos houve pouca coincidência de referencial teórico. Como existe uma grande quantidade de trabalhos sobre o tema 'material didático', é provável que essa abundância leve a não repetição de referencial nos artigos.

Metodologia - neste item, buscou-se saber quais as abordagens utilizadas, quais foram os participantes da pesquisa, que instrumentos foram usados para coletar os dados e como foram 
os mesmos analisados. Segundo Elliot (2011, p. 945):

a Metodologia apresenta os procedimentos utilizados, o que inclui o desenho e a abordagem adotados pela avaliação, entre outros procedimentos. Busca-se saber, neste aspecto, o que é necessário para desenvolver as etapas metodológicas da avaliação e como foram desenvolvidas.

Da leitura dos artigos, verificou-se a predominância (21) de artigos que afirmavam terem seguido uma metodologia qualitativa. Apesar desta afirmativa, observou-se que muitos resultados foram apresentados por meio de estatística descritiva, usada para análise dos dados.

Tabela 5: Metodologia de pesquisa/avaliação

\begin{tabular}{|l|c|}
\hline \multicolumn{1}{|c|}{ Abordagem } & Quantidade de artigos \\
\hline Quantitativa & 4 \\
\hline Qualitativa & 21 \\
\hline Quanti-qualitativa & 1 \\
\hline
\end{tabular}

Fonte: as Autoras (2018)

Observou-se que três artigos não continham o tópico metodologia (ou método ou procedimento metodológico), mas as informações referentes a esta parte estavam descritas com clareza no meio do texto, sendo especificados os procedimentos utilizados no estudo. Já 23 artigos possuíam o tópico metodologia no corpo do texto, sendo que na maior parte dos artigos foi descrito em detalhes; apenas poucos falaram ligeiramente como foi feito o estudo.

No que tange aos tipos de participantes dos estudos, observou-se uma preferência pelos alunos uma vez que os autores estavam preocupados em melhorar a formação dos estudantes através de novos métodos de aprendizagem. Como se pode ver na Tabela 6, alguns artigos usaram mais de um tipo de respondente. Cabe salientar que três artigos analisaram livros didáticos e um fez uma revisão de literatura sobre mapa conceitual. Estes ficaram na categoria 'não tem' respondente.

Tabela 6: Tipo de respondentes/participantes

\begin{tabular}{|l|c|}
\hline Respondentes & Artigos \\
\hline Alunos & 18 \\
\hline Analista de sistema & 1 \\
\hline Docente & 8 \\
\hline Enfermeiros & 3 \\
\hline Especialista & 2 \\
\hline Mães & 1 \\
\hline Produtores rurais & 1 \\
\hline Não tem & 4 \\
\hline
\end{tabular}

Fonte: as Autoras (2018)

Quanto aos instrumentos utilizados, como no caso dos respondentes, vários deles usaram mais de um instrumento, embora tenha havido uma preferência por questionários (13) 
e por roteiro de grupo focal (4). Três artigos não utilizaram instrumentos, porque fizeram uma revisão de literatura sobre o tema pesquisado. Esses resultados aparecem na Tabela 7.

Tabela7: Instrumentos usados na metodologia

\begin{tabular}{|l|c|}
\hline \multicolumn{1}{|c|}{ Instrumentos } & Artigos \\
\hline Escala de Likert & 3 \\
\hline Ficha de avaliação/atividades & 2 \\
\hline Formulário & 2 \\
\hline Roteiro de grupo focal & 4 \\
\hline Roteiro de observação & 2 \\
\hline Questionário & 13 \\
\hline Outros que aparecem 1 vezes & 10 \\
\hline Não tem & 3 \\
\hline
\end{tabular}

Fonte: as Autoras (2018)

Em termos de ilustrações utilizadas nos artigos, verificou-se a predominância de Quadros, como se pode ver na Tabela 8.

Tabela 8: Tipos de ilustrações utilizadas nos artigos

\begin{tabular}{|l|c|}
\hline \multicolumn{1}{|c|}{ Ilustrações } & Artigos \\
\hline Figuras & 5 \\
\hline Gráficos & 5 \\
\hline Quadros & 11 \\
\hline Tabelas & 3 \\
\hline Não tem & 7 \\
\hline
\end{tabular}

Fonte: as Autoras (2018)

Figuras e gráficos foram usados em cinco artigos, as tabelas em três, e sete não utilizaram qualquer tipo de ilustrações. Também se observou que vários artigos se valeram de mais de um tipo de ilustração. Verificou-se que a Tabela 5, que destacou os tipos de abordagem dos estudos, estava coerente com a Tabela 8 (Tipos de ilustrações).

Resultados -todos os artigos têm resultados que podem ser aplicados no ensino. Isso acontece por que os artigos estavam avaliando o desenvolvimento de um produto e sua aplicação na situação de ensino-aprendizagem. Como exemplo, apresenta-se um trecho do artigo de Alvarez (2011, p. 8):

Os resultados do estudo mostraram que o OVADOR propiciou experiência educacional interativa aos estudantes de graduação em enfermagem, semelhante a situações reais vivenciadas na assistência em saúde. Conclui-se que o OVADOR é método dinâmico, construtivo, inovador e atrativo para a aprendizagem dos estudantes, no ensino para avaliação simulada da dor aguda, aplicado a estudantes de enfermagem.

Todos os objetivos indicados nos artigos foram atendidos segundo os resultados das análises dos dados coletados. Em sua maioria, tais dados foram analisados com o uso da 
estatística descritiva, conforme o exemplo que se segue: Quanto à utilização dos objetos educacionais digitais, compostos por seis variáveis, os docentes direcionaram suas respostas para as categorias 'sempre' (70, 5\%) e 'frequentemente' (25,6\%)”. (COGO, 2009, p. 292).

Recomendações - de um modo geral, as recomendações apresentadas nos artigos seguem duas linhas: dar continuidade aos estudos ou apontar melhorias que precisam ser feitas. Observouse que as recomendações foram elaboradas com base nas conclusões dos estudos. Dos 26 artigos selecionados, 13 apresentam apenas uma conclusão; os outros 13, além da conclusão, trazem algum tipo de recomendações. Dois exemplos podem ser citados:

“[...] sugerimos que a pesquisa-ação pode contribuir para a aproximação entre ensino e pesquisa, à medida que os participantes vão alargando os ciclos de ação e reflexão, mantendo como foco as práticas educativas”. (BETTI, 2010, p. 150); e

“[...] faz-se necessário o desenvolvimento de estudos posteriores, tendo como foco o impacto no processo ensino/aprendizagem e a retenção do conhecimento por parte de estudantes de graduação e enfermeiros, na utilização do objeto virtual de aprendizagem”. (GÓES, 2011, p. 7).

Alguns autores não viram necessidade de aprofundar os estudos, porém outros sentiram a importância de desenvolver novos estudos para melhorar os métodos de aprendizagem utilizados. Estes foram justamente os autores que desenvolveram os softwares ou alguma outra tecnologia e que apresentaram as recomendações para dar seguimento aos estudos realizados.

\section{Respondendo à questão do estudo: um parecer avaliativo}

A questão que norteou a construção deste Parecer: em que medida os artigos da categoria 'avaliação de material didático' se integram ao estado da arte da avaliação, organizado pela disciplina Prática de Avaliação, exige, de início, que se recorra ao campo conceitual que aborda o que é um Estado da Arte. O Estado da Arte é uma pesquisa de natureza bibliográfica, que constitui extenso levantamento bibliográfico, podendo ser real ou virtual. Ele procura:

mapear e discutir uma certa produção acadêmica em diferentes campos do conhecimento, tentando responder que aspectos e dimensões vem sendo destacados e privilegiados em diferentes épocas e lugares. [...] também são conhecidos por realizar uma metodologia de caráter inventariante e descritivo da produção acadêmica e cientifica sobre o tema que busca investigar. (FERREIRA, 2002, p. 258)

Teixeira (2006, p, 60) complementa, dizendo que o Estado da Arte "procura compreender o conhecimento elaborado, acumulado e sistematizado sobre determinado tema, num período temporal que, além de resgatar, condensa a produção acadêmica numa área de 
conhecimento específico”.

A partir desses conceitos, pode-se visualizar a relação entre um Estado da Arte em Avaliação e um Estado da Arte em Avaliação de Material Didático. Trata-se de uma relação natural de dependência, sendo um mais abrangente e o outro mais restrito, mas ambos se interconectam pela existência de uma palavra que permeia os dois: Avaliação. Essa relação pode ser observada quando se analisa os diferentes itens que normalmente compõem um artigo, como situação problema, objetivos, metodologia e resultados.

Em todos os artigos, a apresentação de uma situação problema estava voltada para a avaliação de algum objeto e este se relacionava com um problema, ou uma necessidade, que demandava uma solução, tendo como suporte uma abordagem metodológica de pesquisa qualitativa ou quantitativa.

No que tange aos objetivos, foi observada a relevância da avaliação a ser conduzida, visando solucionar o problema ou necessidade apontada. Na metodologia verificou-se o uso de abordagens qualitativa e quantitativa para responder à avaliação dos objetivos propostos, tendo sido descritos o passo a passo usado para avaliar. Cabe chamar atenção que diversos artigos, apesar de indicarem que estavam usando uma metodologia qualitativa, na realidade, usaram estatística descritiva na análise dos dados o que fez com se filiassem à abordagem quantitativa. Os resultados apresentados foram positivos, pois ficou evidente que os objetivos dos estudos foram atingidos.

Os artigos contêm referenciais teóricos que embasam sua linha de raciocínio e em geral, os resultados encontrados são passíveis de serem colocados em prática. Desse modo, pode-se dizer que os objetivos propostos para os estudos foram alcançados. Recomendações ou sugestões foram encontradas somente em 13 artigos, mas todos possuíam uma conclusão do trabalho realizado.

Pode-se, então, afirmar que: (a) todos os 26 artigos apresentaram uma situação problemática; (b) 22 artigos estavam com seus objetivos claramente definidos, o demais, quatro, tiveram seus objetivos inferidos por meio de (re)leitura do artigo; (c) todos possuíam referencial teórico vinculado ao material didático escolhido para avaliar: (d) na metodologia a abordagem privilegiada foi a qualitativa (21 artigos), os demais foram quatro com abordagem quantitativa e um com abordagem quanti-qualitativa; (e) todos tinham resultados; e (f) todos apresentaram conclusões, sendo que, destes, 13 indicaram recomendações.

Conclui-se, então, que a maior parte dos artigos apresentou uma estruturação adequada ao que se define como 'artigo acadêmico'. A análise dos 26 artigos da base de dados e-Aval, referentes à categoria Avaliação de Material Didático, entre os anos de 2001 a 2016, permitiu o desenvolvimento de um recorte do Estado da Arte da Avaliação. 


\section{Referências}

ADELINO, Paula Resende; FONSECA, Maria da Conceição Ferreira Reis. Matemática e texto: práticas de numeramento num livro didático da educação de pessoas jovens e adultas. Revista Brasileira de Educação, Rio de Janeiro, v. 19, n. 56, jan/mar. 2014, p. 181-200. Disponível em: <http://www.scielo.br/scielo.php?script=sci_arttext\&pid=S141324782014000100010>. Acesso em: 20 fev. 2018.

ALVAREZ, Ana Graziela; SASSO, Grace Teresinha Marcon Dal. Aplicação de objeto virtual de aprendizagem, para avaliação simulada de dor aguda, em estudantes de enfermagem. Revista Latino-Americana de Enfermagem, São Paulo, v. 19, n. 2, mar/abr. 2011, p. 229-237. Disponível em:<http://www.scielo.br/scielo.php?script=sci_abstract\&pid=So10411692011000200002 \&lng=pt\&nrm=iso\&tlng=pt>. Acesso em: 20 fev. 2018.

BETTI, Mauro. Imagens em avalia-ação: uma pesquisa-ação sobre o uso de matérias televisivas em aulas de educação física. Educar em Revista, Curitiba, n. especial 2, 2010, p. 137-152. Disponível em: <http://www.scielo.br/scielo.php?script=sci_arttext\&pid=So10440602010000500008\&lng=pt\&nrm=iso\&tlng=pt>. Acesso em: 20 fev. 2018.

COGO, Ana Luísa Petersen et al. Objetos educacionais digitais em enfermagem: avaliação por docentes de um curso de graduação. Revista da Escola de Enfermagem da USP, São Paulo, v. 43, n. 2, 2009, p. 295-299. Disponível em: <http://www.scielo.br/scielo.php?script=sci_abstract\&pid=Soo8062342009000200006\&lng=pt\&nrm=iso\&tlng=pt>. Acesso em: 20 fev. 2018.

ELLIOT, Ligia Gomes. Meta-avaliação: das abordagens às possibilidades de aplicação. Ensaio: aval. Pol. Públ. Educ., Rio de Janeiro, v. 19, n. 73, out/dez. 2011, p. 941-964. Disponível em: <http://www.scielo.br/pdf/ensaio/v19n73/11.pdf>. Acesso em: 2 abril 2018.

FERREIRA, Norma Sandra de Almeida. As pesquisas denominadas "estado da arte". Educação \& Sociedade, ano XXIII, n. 79, agosto, 2002, p. 257-272. Disponível em: http://www.scielo.br/pdf/es/v23n79/10857.pdf Acesso em: 15 abril 2018.

FACULDADE CESGRANRIO, E-AVAL. O estado da arte da área da Avaliação. Relatório técnico 2018. Rio de Janeiro: Fundação Cesgranrio, 2018. 
GÓES, Fernanda dos Santos Nogueira de et al. Avaliação do objeto virtual de aprendizagem "Raciocínio diagnóstico em enfermagem aplicado ao prematuro”. Revista Latino-Americana de Enfermagem, São Paulo, v. 19, n. 4, jul/ago. 2011, p. 894-901. Disponível em: <http://www.scielo.br/scielo.php?script=sci_abstract\&pid=S010411692011000400007\&lng=pt\&nrm=iso\&tlng=pt>. Acesso em: 20 fev. 2018.

JULIANI, Carmen Maria Casquel Monti; KURCGANT, Paulina. Tecnologia educacional: avaliação de um website sobre Escala de Pessoal de Enfermagem. Revista da Escola de Enfermagem da USP, São Paulo, v. 43, n. 3, 2009, p. 512519.

Disponível

em: <http://www.scielo.br/scielo.php?script=sci_abstract\&pid=Soo8o62342009000300003\&lng=pt\&nrm=iso\&tlng=pt > . Acesso em: 20 fev. 2018. MANCEBO, Deise. Trabalho docente e produção de conhecimento. Psicologia \& Sociedade, Rio de Janeiro, v. 25, n. 3, 2013, p. 519-526. Disponível em: <http://www.scielo.br/pdf/psoc/v25n3/o6.pdf>. Acesso: 17 mar. 2018.

TEIXEIRA, Célia Regina. O "Estado da Arte": a concepção de avaliação educacional veiculada na produção acadêmica do Programa de Pós-Graduação em Educação: 1975-200o. Cadernos de Pós-Graduação - educação, São Paulo, v. 5, n. 1, p. 59-66, 2006. Disponível em: http://docplayer.com.br/63463997Celia-regina-teixeira-doutora-em-educacao-puc-sp-docente-da-uninove-saopaulo-sp-brasil.html Aceso 18 abril 2018

PIMENTA, Denise Nacif et al. A importância do ergo design na avaliação de cd-rom sobre dengue e doença de chagas na educação em saúde. Trabalho, Educação e Saúde, Rio de Janeiro, v. 4, n. 1, 2008., p.147-168. Disponível em: <http://www.scielo.br/scielo.php?script=sci_arttext\&pid=S1981$77462008000100008 \& \operatorname{lng}=$ pt\&tlng=pt $>$. Acesso em: 20 fev. 2018.

PINTO, Vladimir Camelo et al. Avaliação de um programa para computador de mão no auxílio ao ensino de oftalmologia para estudantes de medicina. Revista Brasileira de Oftalmologia, Bahia, v.69, n. 6, 2010, p. 352-36o. Disponível em: <http://www.scielo.br/scielo.php?script=sci_abstract\&pid=So03472802010000600002\&lng=pt\&nrm=iso\&tlng=pt>. Acesso em: 20 fev. 2018. ZEM-MASCARENHA, Silvia Helena; CASSIANI, Silvia Helena De Bortoli. Desenvolvimento e avaliação de um software educacional para o ensino de 
enfermagem pediátrica. Revista Latino-Americana de Enfermagem, São Paulo, v. 9, n. 6, nov./dez. 2001, p. 13-18. Disponível em: $<$ http://www.scielo.br/scielo.php?script=sci_arttext\&pid=So10411692001000600003 \&lng=pt\&tlng=pt >. Acesso em: 20 fev. 2018.

\section{Biografia Resumida}

Lúcia Regina Goulart Vilarinho: Pedagoga (PUC - Rio), Mestrado e Doutorado em Educação (UFRJ), docente aposentada da Faculdade de Educação da UFRJ, docente do PPGE da Universidade Estácio de Sá, atualmente docente do Mestrado Profissional em Avaliação da Fundação Cesgranrio.

\section{Link do Lattes:}

http://buscatextual.cnpq.br/buscatextual/visualizacv.do?id=K4727186D6

Contato: luciagvilarinho@gmail.com

Kátia Taucei Perez - Graduada em Biblioteconomia pela Universidade Santa Úrsula / RJ, Mestre em Avaliação pela Faculdade Cesgranrio. Bibliotecária da Escola de Belas Artes da UFRJ.

\section{Link do Lattes:}

http://buscatextual.cnpq.br/buscatextual/visualizacv.do?id=K8081437P9

Contato:ktaucei@yahoo.com.br

Sandra Maria Martins Redoválio Ferreira: Graduada em Psicologia, Mestre em Avaliação pela Faculdade Cesgranrio, docente do ensino de graduação da Faculdade Cesgranrio.

Link do Lattes: http://lattes.cnpq.br/7928378322641468

Contato: sandramartins36@gmail.com 